\title{
Comparison of single and double cladding long period fiber grating sensor using an intensity modulation interrogation system
}

\author{
Chow-Shing Shin ${ }^{\mathrm{a}, *}$, Chia-Chin Chiang ${ }^{\mathrm{a}}$, Shien-Kuei Liaw ${ }^{\mathrm{b}}$ \\ ${ }^{a}$ Department of Mechanical Engineering, National Taiwan University, No. 1, Sec. 4, Roosevelt Road, Taipei 10617, Taiwan \\ ${ }^{\mathrm{b}}$ Department of Electron Engineering, National Taiwan University of Science and Technology, No. 43, Sec. 4, \\ Keelung Road, Taipei 10617, Taiwan
}

Received 26 April 2005; received in revised form 23 June 2005; accepted 20 July 2005

\begin{abstract}
The performance of long period fiber grating (LPFG) sensors written in single cladding and double cladding fibers have been compared by using a fast responding interrogation system based on intensity modulation. Temperature and dynamic strain monitoring using this system have been demonstrated. This system is capable of resolving strain to 0.2 and $0.4 \mu \varepsilon$ at a loading frequency of $20 \mathrm{~Hz}$, and temperature resolution to 0.02 and $0.19^{\circ} \mathrm{C}$ by using $\mathrm{LPFG}$ in the single cladding (SC-LPFG) and double cladding (DC-LPFG), respectively.
\end{abstract}

(C) 2005 Elsevier B.V. All rights reserved.

Keywords: Fiber Bragg gratings; Long period fiber grating; Optical fiber sensor; Intensity modulation

\section{Introduction}

Long period fiber grating (LPFG) has found increasing applications in optical communication and sensing systems. LPFG promotes the coupling between the propagating core modes and copropagating cladding modes. In optical communications, LPFG devices have been demonstrated for applications such as in band-rejection filters

\footnotetext{
* Corresponding author. Tel./fax: +886 223622160.

E-mail address: csshin@ntu.edu.tw (C.-S. Shin).
}

[1], gain-flattening filters [2]. In the sensing applications, LPFG plays an important role as in sensing for temperature [3-6], strain [4,5], and refractive index [7,8]. These sensors possess a number of advantages over conventional sensors. For example, they are light and small in diameter yet they have good sensitivity and good long-term stability. They are also relatively free from corrosion attack [9], and electromagnetic interferences $[4,5]$ that seriously affect many conventional sensors. Physical quantity changes are reflected as wavelength shift in the characteristic resonant 
dip in the transmitted spectrum of LPFG. LPFG were often written in single cladding (SC) fiber. It has been shown that the coupling strength of a LPFG decreased so that the resonant dip vanished at certain refractive indices [10]. This will create problems for applications where the sensor has to be embedded in a medium such as a polymeric composite structure. A number of works have pointed out the environmental refractive index sensitivity may be greatly reduced by fabricating LPFG in double cladding fibers [11-14]. These works mainly focused on analyzing the spectral sensitivities of LPFG in double cladding fibers in response to temperature variations and bending $[13,14]$. Similar investigation on strain sensitivity is more limited [14] and has been limited to quasi-static strain variations to the best of our knowledge. In structural applications, dynamic strain monitoring is a common requirement. In the current work, the characteristics of LPFG in double cladding fiber has been studied and comparison has been made with that in single cladding fiber.

\section{Experimental set-up}

LPFGs were fabricated in a single cladding (SC) and double cladding (DC) photosensitive fibers. The single cladding fiber is $\mathrm{Ge}-\mathrm{B}$ co-doped and refractive indices of 1.458 in the core and 1.446 in the cladding. The diameters of the core and cladding are 9 and $125 \mu \mathrm{m}$, respectively. The double cladding fiber is $\mathrm{Ge}-\mathrm{P}$ co-doped with different dope concentrations to give refractive indices of 1.4530 in the core and 1.4459 in the inner cladding. The outer cladding is made of pure Silicon dioxide with a refractive index of 1.4443 . The diameters of the core, inner cladding, and outer cladding are $9,55,125 \mu \mathrm{m}$, respectively. LPFGs were fabricated by exposing them to a $248-\mathrm{nm}$ $\mathrm{UV}$ radiation from an $\mathrm{KrF}$ Excimer laser through an amplitude mask (period $400 \mu \mathrm{m}$ ). Along the fiber core, the LPFG has a periodic refractive index modulation of $200 \mu \mathrm{m}$ for per pitch. The dip losses of the SC-LPFG and the DC-LPFG were about 12 and $15 \mathrm{~dB}$ at 1569 and $1571 \mathrm{~nm}$, respectively. These correspond to $94 \%$ and $97 \%$ of power ratio being coupled to cladding in the SC-LPFG and DC-LPFG, respectively.

For temperature measurement, the fiber sensor was put into a temperature control chamber and a thermo-coupler is located beside the fiber sensor to record the instantaneous temperature.

For strain measurement, the fiber sensors were attached to the surface of a 16-layer quasi-isotropic carbon fiber epoxy laminate specimen. The specimen geometry conformed to ASTM D3039 [15] for tensile testing of fiber reinforced composites. A strain gauge was also attached to the opposite surface of the composite specimen. The specimen is loaded in a servo-hydraulic testing machine with a cyclic sinusoidal waveform which amplitude is $2000 \mu \varepsilon$ at $20 \mathrm{~Hz}$.

In the previous studies, LPFG shift has commonly been measured by diffraction grating technology such as using an optical spectrum analyzer (OSA) [11-14]. This technique is too slow for dynamic measurement. In the current work, an all fiber interrogating system (Fig. 1) taking advantage of the edge filtering effect of a LPFG [16,17] has been employed. The system was originally proposed to interrogate FBG sensors [16] and measurement up to $150 \mathrm{kHz}$ has been demonstrated [17]. To the best of our knowledge, interrogation of an LPFG using the fiber laser has not been attempted and more importantly, the dynamic response of LPFG is still unclear. In this study, we employ such a system to investigate the dynamic response of LPFG. The FBG and the gain medium of Er-doped fiber forms a lasing cavity [18] which pumps laser light at the Bragg wavelength through to the LPFG. As the LPFG responds to the measurand, the resonant dip shifts, thus edge filtering the intensity of light energy to different degrees. This energy may be measured with an InGaAs photodetector circuitry to reflect the measurand. The FBG involved was also fabricated in the Ge-B co-doped single cladding fiber by side writing using a phase mask with a period of $1.08 \mu \mathrm{m}$. This resulted in a peak wavelength of $\sim 1564 \mathrm{~nm}$ to match with the dip spectrum of the LPFGs in order to achieve the edge filtering effect. The reflectivity of the resulting FBG was about 99\% and the FWHM (full width at half maximum) of the FBG is about $0.175 \mathrm{~nm}$. 


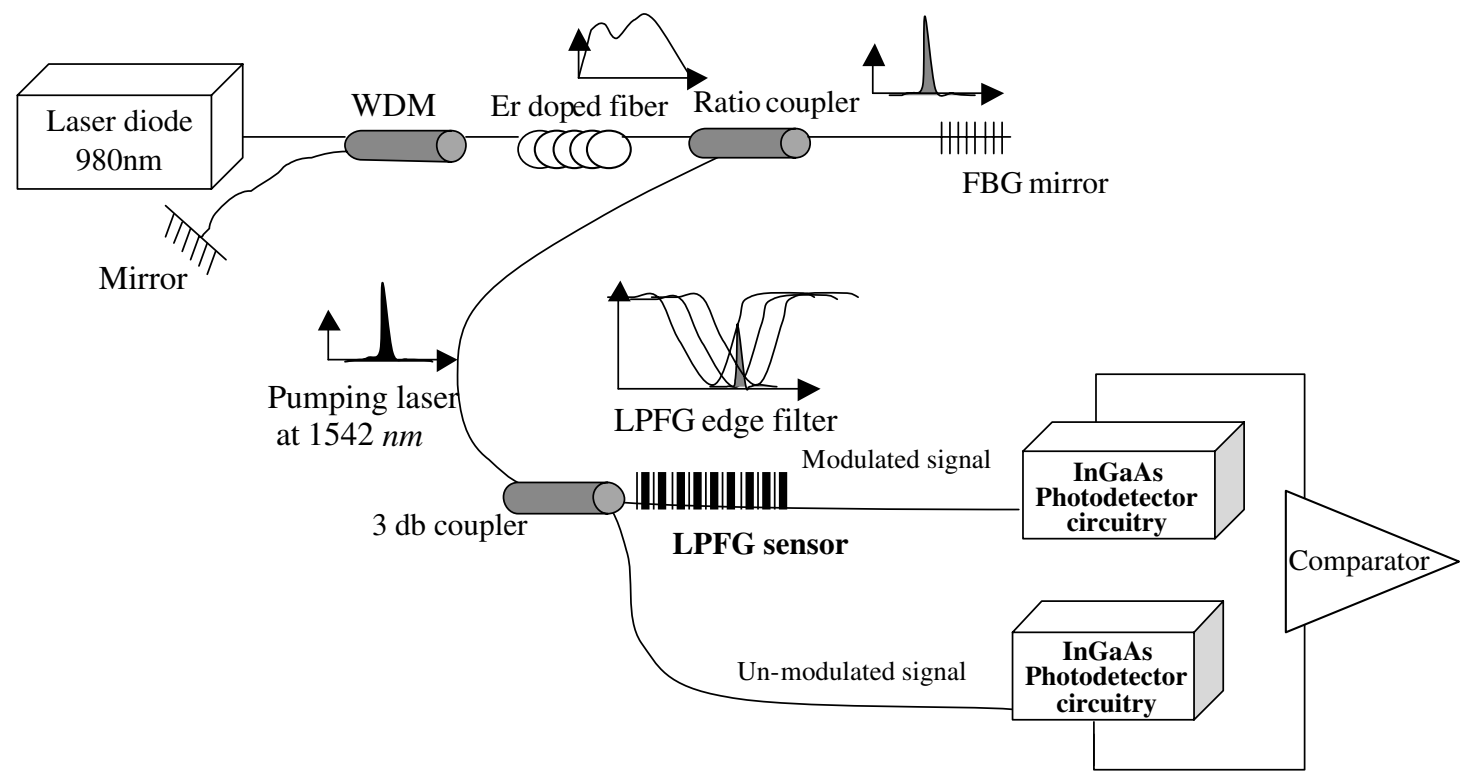

Fig. 1. Schematic diagram of the hybrid FBG-LPFG intensity modulation sensing system used for interrogating the measured parameters.

\section{Experimental results and discussion}

\subsection{Basic properties of $L P F G$ sensors}

In a long period fiber grating, the phase-matching condition [5] is

$\lambda=\left[n_{\mathrm{eff}}-n_{\mathrm{cl}}^{i}\right] \Lambda$,

where $\lambda$ is the central wavelength of the resonant dip spectrum, $\Lambda$ is the periodicity of the grating, $n_{\text {eff }}$ is the effective refractive index of the core mode, $n_{\mathrm{cl}}^{i}$ is the effective refractive index of the cladding mode, It is clear from Eq. (1) that the resonance wavelength is not only related to the refractive index $\left(n_{\text {eff }}\right)$ of the core mode, but also to the refractive index $\left(n_{\mathrm{cl}}^{i}\right)$ of the $i$ th cladding mode, which be dependent on the indices of various fiber cladding layers. When phasematching condition was satisfied, the core mode will couple to the cladding mode and part of the spectrum will be attenuated, which may result in a dip in the transmitted spectrum. The cladding mode will interact with material surrounding the cladding. Changes optical properties of the ambient material will result in a change in the dip spectrum $[4,5]$.
Wavelength drift due to temperature sensitivity is obtained by differentiating Eq. (1) with respect to temperature $T$ [5]

$\frac{\mathrm{d} \lambda}{\mathrm{d} T}=\frac{\mathrm{d} \lambda}{\mathrm{d}\left(\delta n_{\mathrm{eff}}\right)}\left(\frac{\mathrm{d} n_{\mathrm{eff}}}{\mathrm{d} T}-\frac{\mathrm{d} n_{\mathrm{cl}}^{i}}{\mathrm{~d} T}\right)+\Lambda \frac{\mathrm{d} \lambda}{\mathrm{d} \Lambda} \frac{1}{L} \frac{\mathrm{d} L}{\mathrm{~d} T}$,

where $T$ is the temperature and $L$ is the length of the LPFG. The first term on the right-hand side of Eq. (2) is a material factor, and is related to the change in the differential refractive index of the core and cladding arising from the thermooptic effect. The term $\mathrm{d} n_{\mathrm{cl}}^{i} / \mathrm{d} T$ is the thermo-optic coefficient of the $i$ th cladding mode, which in turn can be calculated approximated from the thermooptic coefficient of the cladding layer material containing the majority of the energy of the $i$ th cladding mode. The second term is the waveguide factor as it results from variation of the LPFG's period. The magnitude and sign of the term depend upon the order of the cladding mode.

The axial strain sensitivity of LPFGs may be assessed by differentiating Eq. (1) with respect to strain $\varepsilon[5]$

$\frac{\mathrm{d} \lambda}{\mathrm{d} \varepsilon}=\frac{\mathrm{d} \lambda}{\mathrm{d}\left(\delta n_{\mathrm{eff}}\right)}\left(\frac{\mathrm{d} n_{\mathrm{eff}}}{\mathrm{d} \varepsilon}-\frac{\mathrm{d} n_{\mathrm{cl}}^{i}}{\mathrm{~d} \varepsilon}\right)+\Lambda \frac{\mathrm{d} \lambda}{\mathrm{d} \Lambda}$. 
Again, the sensitivity comprises a material influence and a waveguide effect. Material influence comes from the change in fiber dimensions and the strain-optic effect. The term $\mathrm{d} n_{\mathrm{cl}}^{i} / \mathrm{d} T$ is the strain-optic coefficient of $i$ th the cladding mode, which may be taken as the strain-optic coefficient of the cladding layer in which that mode is predominantly guided. The waveguide effect also arises from the slope of the dispersion term $\mathrm{d} \lambda / \mathrm{d} \Lambda$ as above.

The thermo-optic coefficient $\left(\mathrm{d} n_{\mathrm{cl}}^{i} / \mathrm{d} T\right)$ and the strain-optic coefficients $\left(\mathrm{d} n_{\mathrm{cl}}^{i} / \mathrm{d} \varepsilon\right)$ in Eqs. (2) and (3) of a glass material will vary with the composition and concentration of dopants in the different fiber cladding layers. These result in the different sensitivity and character between SC-LPFG and DC-LPFG.

\subsection{Environment effect of $S C-L P F G$ and $D C-L P F G$}

\subsubsection{Environmental index effect}

Changes in the optical properties of the ambient material will be reflected as a change in the resonant dip spectrum of LFBG [4,5]. Fig. 2 shows the resulting spectra coming through a SC-LPFG when the fiber is put into a media with various refractive indices. It can be seen that when the fiber is surrounded by a medium with a refractive index of 1.47 , and the characteristic resonant dip of this LPFG will disappear. Also, no dip spectrum was observed when the SC-LPFG is embedded in the

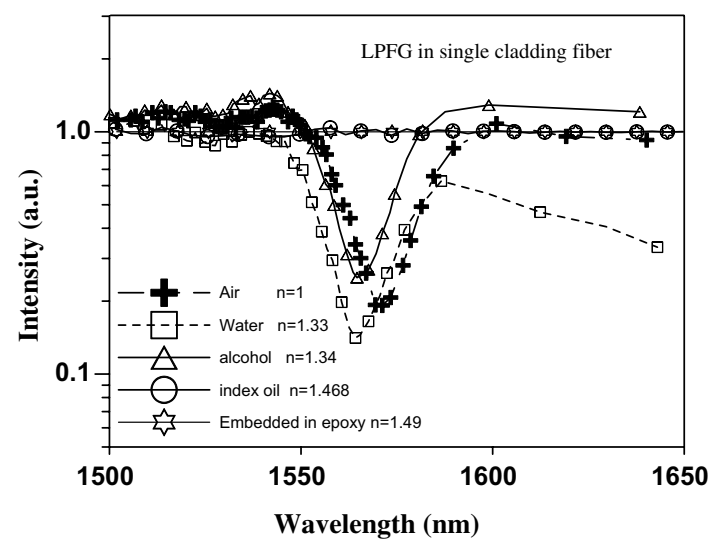

Fig. 2. Transmitted spectra through the SC-LPFG when the latter is submerged in media with different refractive indices. carbon fiber epoxy composite. It has been point out that when the refractive index of the external medium is in the range of 1.45-1.64, the coupling strength of a LPFG is decreased so that the resonant dip vanished [10]. When a fiber sensor is used to measure the physical quantities such as strain or temperature on a mechanical structure, often it has to be stuck onto the surface or even be embedded in the structure. In fact, being embeddable is one of the major attractiveness of the in-fiber sensors in smart structures. However, common polymeric adhesives for sensor fixation and the resin matrix of fiber reinforce composites for sensor embedment have refractive indices near 1.5. This will render the LPFG useless since the embedded sensors are used in most structural applications.

By using the DC-LPFG, the cladding mode is confined in the inner cladding. This can effectively isolates the interaction between the cladding mode and the ambient environment. Fig. 3 shows the resulting spectra through the DC-LPFG that has been put into different media. It is clear that the characteristic resonant dips still exist despite of being embedded in a widely used carbon fiber epoxy composite. The intensity of the spectrum through the embedded LPFG is somewhat lower than that of the freely hanging fiber. This degradation may cause the composite curing process, which exposed the fiber to a pressure of 0.69 $\mathrm{MPa}$ at $120^{\circ} \mathrm{C}$ for half an hour. The spectrum of the embedded LPFG has also being shifted presumably due to the residual stresses in the cured

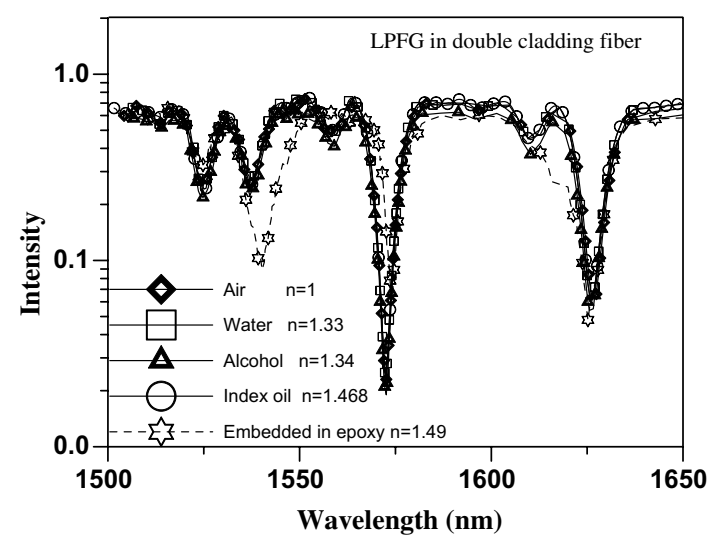

Fig. 3. Transmitted spectra through the DC-LPFG when the latter is submerged in media with different refractive indices. 
composite, so that the embedded LPFG is actually under some degree of straining.

\subsubsection{Temperature effect}

Temperature calibration using an OSA shows that the temperature coefficients of the SC-PLG and DC-PLG are -284 and $29.7 \mathrm{pm} /{ }^{\circ} \mathrm{C}$, respectively. Fig. 4 shows the temperature induced variation interrogated by the intensity modulation sensing system with the SC-LPFG and the DCLPFG. Temperature resolution of 0.02 and $0.19^{\circ} \mathrm{C}$ were obtained, respectively. However, the relation between temperature and voltage output for the SC-LPFG is not one-to-one. It is because that modulation by edge filtering can take place on both sides of the resonant dip. The current system could not discriminate on which side the Bragg wavelength lies relative to the dip. As a result, only half of the resonant dip can be used for energy modulation in practice. Thus, this usable temperature range will quickly be exhausted. This range can be considerably increased at the expense of resolution degrade. Also, the DC-LPFG has linear relationship between the modulated energy output and temperature under an applicable temperature range. The temperature resolution is 2.5 times more precise than that in [5].

Since these fiber sensor systems are small in size, it will take up minimal thermal energy from the object. Moreover, they are embedded into a structure to allow interior temperature of an object to

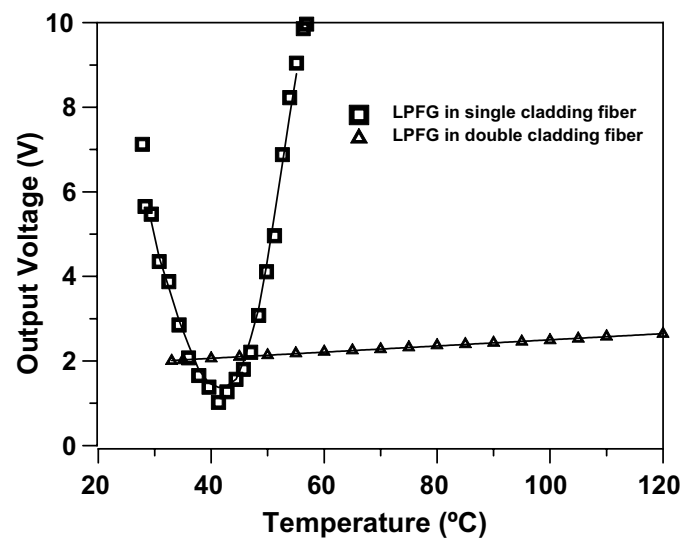

Fig. 4. Temperature induced wavelength shift interrogated by using the intensity modulation sensing system. be measured. These properties, as well as their applicable range and resolution, give them significant advantages over many conventional temperature sensors.

\subsection{Strain measurement}

Calibration with applied strain shows its relation to wavelength shift is quite linear. And the strain coefficients are $1.59 \mathrm{pm} / \mu \varepsilon$ for the SCLPFG and $0.8 \mathrm{pm} / \mu \varepsilon$ for the DC-LPFG. With a data acquisition resolution of $0.2 \mathrm{mV}$, these correspond to strain resolutions of 0.2 and $0.4 \mu \varepsilon$, respectively. Fig. 5(a) and (b) shows the measured results from the current system when the same dynamic strain as above is applied to
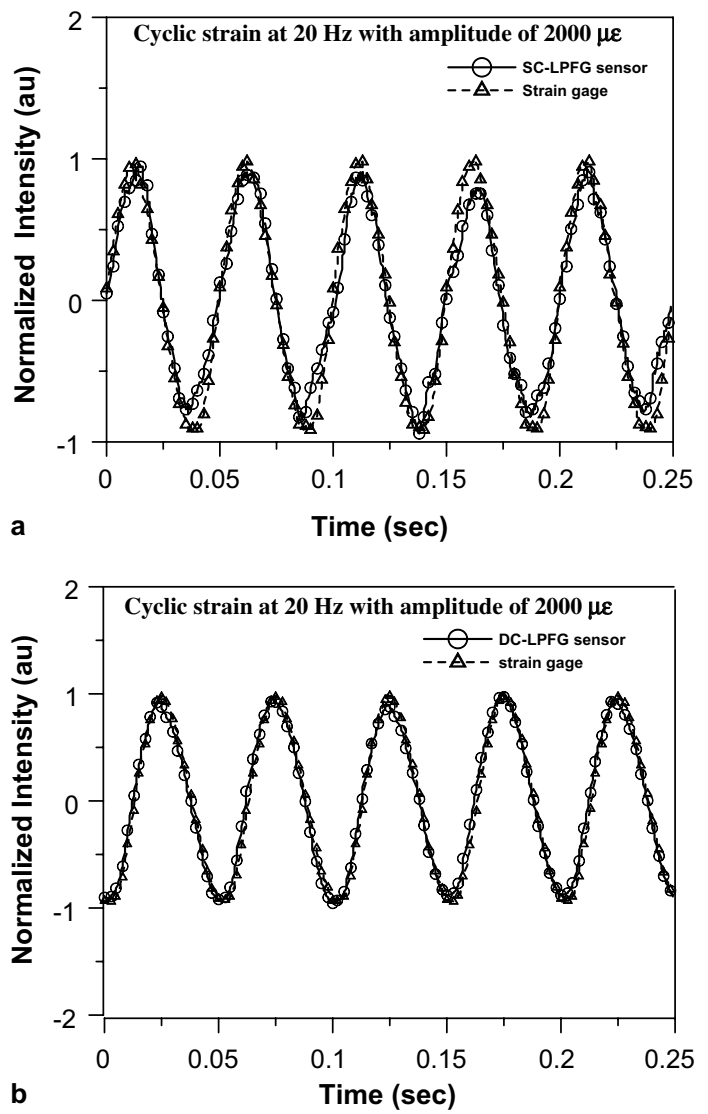

Fig. 5. Comparison of the sinusoidal strain variation measured by the intensity modulation sensing system using (a) SC-LPFG or (b) DC-LPFG with the conventional strain gage output. 
Table 1

Temperature and strain coefficients of different LPFG

\begin{tabular}{lll}
\hline $\begin{array}{l}\text { Fiber type housing } \\
\text { the LPFG }\end{array}$ & $\begin{array}{l}\text { Temperature } \\
\text { coefficient }\left(\mathrm{pm} /{ }^{\circ} \mathrm{C}\right)\end{array}$ & $\begin{array}{l}\text { Strain coefficient } \\
(\mathrm{pm} / \mu \varepsilon)\end{array}$ \\
\hline Single cladding & -284 & 1.59 \\
Double cladding & 29.7 & 0.8 \\
\hline
\end{tabular}

the SC-LPFG and DC-LPFG. For the SC-LPFG shows in Fig. 5(a), the signal exhibited occasional spikes and fluctuation in the peaks and valleys. However, the output from the strain gauge remained fairly constant, indicating that the applied strain was quite steady and free from spikes. The discrepancy may be due to the high temperature-sensitivity of the SC-LPFG. On the other hand, Fig. 5(b) shows that the measured results for the DC-LPFG is virtually the same as that given by the strain gauge.

\subsection{Comparison of SC-LPFG and DC-LPFG sensors}

Table 1 shows the strain and temperature coefficients of SC-LPFG and the DC-LPFG. The temperature coefficient of the SC-LPFG is negative and is about an order of magnitude larger than that of the DC-LPFG. Its strain coefficient is double that of the DC-LPFG. Such large temperature coefficient is advantages in providing a good temperature resolution but on the down side, it limits the applicable temperature measurement range. The DC-LPFG has a good balance between the temperature sensitivity and measurement range. Though the DC-LPFG has only half the strain sensitivity than that of the SC-LPFG, it gives more stable performance in the monitoring of dynamic strain variations.

\section{Conclusions}

A hybrid FBG-LPFG arrangement to achieve intensity modulation has been developed for interrogating wavelength shift in LPFG housed in single and double cladding fibers. It is capable of resolving strain to 0.2 and $0.4 \mu \varepsilon$ at a loading frequency of $20 \mathrm{~Hz}$, and temperature resolution to
0.02 and $0.19^{\circ} \mathrm{C}$, by using the SC-LPFG and DC-LPFG, respectively. The SC-LPFG has a large temperature coefficient $\left(-284 \mathrm{pm} /{ }^{\circ} \mathrm{C}\right)$ and is better fitted for monitoring a small temperature variation over a narrow range. The DC-LPFG has a good balance between temperature sensitivity $\left(29.7 \mathrm{pm} /{ }^{\circ} \mathrm{C}\right)$ and the measurement range. Though the DC-LPFG has only half of the strain sensitivity $(0.8 \mathrm{pm} / \mu \varepsilon)$ than that of the SC-LPFG did, it gives a more stable performance in monitoring the dynamic strain variations. Moreover, the SC-LPFG will lose its characteristic resonant dip spectrum when it is embedded in a carbon fiber epoxy composite while the dip spectrum of DCLPFG is not affected by the embedment. Thus, the DC-LPFG is better suited for applications such as structural integrity monitoring that involves embedment of fiber in the structure and dynamic strain variations.

\section{Acknowledgments}

The work is supported by projects NSC 902212-E-002-175 and NSC 91-2212-E-002-056, Taiwan. We thank the optoelectronic laboratory of NTUST for supporting the KrF Excimer laser.

\section{References}

[1] A.M. Vengsarkar, P.J. Lemaire, J.B. Judkins, V. Bhatia, T. Erdogan, J.E. Sipe, Journal of Lightwave Technology 14 (1996) 58.

[2] A.M. Vengsarkar, P.J. Lemaire, G. Jacobovitz-Veselka, V. Bhatia, J.B. Judkins, in: Proceedings of IOOC'95, PD1-2, Hong Kong, June, 1995.

[3] B.H. Lee, J. Nishii, Proceedings of SPIE - The International Society for Optical Engineering 3746 (1999) 418.

[4] J.A. Besley, T. Wang, L. Reekie, Journal of Lightwave Technology 21 (3) (2003) 848.

[5] S.W. James, R.P. Tatam, Measurement Science and Technology 14 (5) (2003) 49.

[6] K.J. Han, Y.W. Lee, J. Kwon, S. Roh, J. Jung, B. Lee, IEEE Photonics Technology Letters 16 (9) (2004) 2114.

[7] S. Khaliq, S. James, W. Stephen, R.P. Tatam, Optics Letters 26 (16) (2001) 1224.

[8] M.N. Ng, Z. Chen, K.S. Chiang, IEEE Photonics Technology Letters 14 (3) (2002) 361. 
[9] K.R. Cooper, J. Elster, M. Jones, R.G. Kelly, in: IEEE Systems Readiness Technology Conference, 2001, p. 847.

[10] H.J. Patrick, A.D. Kersey, F. Bucholtz, Journal of Lightwave Technology 16 (1998) 1606.

[11] B.A.L. Gwandu, X. Shu, T.D.P. Allsop, W. Zhang, L. Zhang, I. Bennion, Electronics Letters 38 (2002) 695.

[12] T. Allsop, D.J. Webb, I. Bennion, Journal of Lightwave Technology 21 (2003) 264.

[13] T. Allsop, D.J. Webb, I. Bennion, Electronics Letters 39 (2003) 508.
[14] T. Allsop, D.J. Webb, I. Bennion, Optical Fibre Technique 9 (2003) 210.

[15] D3039-76, ASTM Standards and Literature References for Composite Materials, American Society for Testing and Materials, Philadelphia, PA, 1990.

[16] R.W. Fallon, L. Zhang, L.A. Everall, I. Bennion, Measurement Science \& Technology 9 (1998) 1969.

[17] C.S. Shin, C.C. Chiang, Journal of the Chinese Institute of Engineers (in press).

[18] A.D. Kersey, W.W. Morey, Electronics Letters 29 (1993) 112. 